\title{
Neurodynamic measures of functional connectivity and cognition
}

\author{
Selma Supek $\cdot$ Ratko Magjarevic
}

Received: 3 April 2011/Accepted: 7 April 2011/Published online: 22 April 2011

(C) International Federation for Medical and Biological Engineering 2011

Exploring the structure and function of the human brain relies on a range of non-invasive measures and computational approaches. Neurodynamic functional brain imaging methods offer unique insight into the human brain processes by capturing neuronal activity in real time. Measurements of scalp potential differences of spontaneous and evoked activity were the first electrophysiological measure that revealed differences in the measured waveforms which were state, stimulus, task, context etc. dependant. Four decades ago the application of ultrasensitive superconducting quantum interference device (SQUID) for the measurements of extremely week magnetic fields of the human brain led to a dynamic development of magnetoecephalography (MEG) research [2]. Development of this new functional brain imaging method resulted in opening new questions and furthering the analysis of the EEG/ERP as well since the interest of MEG researchers moved from the analysis based on the evaluations of the measured waveforms to explore the approaches to identify and track cortical activations in space and time. Spatio-temporal source localization models and inverse bio-electro-magnetic problem estimation approaches were developed to estimate the underlying neuronal substrates of the evoked activity related to sensory perception, movement, and cognition. Exploration of the underlying dynamic cortical networks of the working human brain was made possible

S. Supek $(\square)$

Department of Physics, Faculty of Science, University of

Zagreb, Bijenicka c. 32, 10000 Zagreb, Croatia

e-mail: selma@phy.hr

R. Magjarevic

Department of Electronic Systems and Information Processing, Faculty of Electrical Engineering and Computing, University of Zagreb, Unska 3, 10000 Zagreb, Croatia not only in the measurement space but also at the functional source level.

During the 1990s, The Decade of the Brain, non-invasive structural and functional brain imaging methods were regarded as revolutionary in exploring the structure and function of the human brain in healthy and diseased states. The wealth of data regarding sensory, motor, and cognitive processes provided initially by positron emission tomography (PET) is dominated during the last two decades by an explosion of research using functional magnetic resonance imaging (fMRI). fMRI is often reported to offer superb millimeter spatial resolution, almost uniform throughout the brain. EEG and MEG were, however, regarded as model dependent and consequently prone to erroneous source location estimation. Multi-modal integration appeared in mid 1990' as a solution to overcome ill-posed nature of the bio-electro-magnetic problem and a low, $2 \mathrm{~s}$ and even $40 \mathrm{~s}$, temporal resolutions of fMRI and PET, respectively. The initial confidence that PET or fMRI identified functional foci could be used to constrain EEG or MEG solution was soon reconsidered.

Unlike in EEG and MEG, there is a great degree of standardization regarding preprocessing and statistical approaches in fMRI. During a rather intensive data gathering period since its discovery important questions such as the reliability of the fMRI results remain almost unconsidered and unanswered [3]. Another open question is the relationship of the hemodynamic and/or metabolic response and the neuronal activity of interest. Despite a widely used terminology referring to PET and fMRI data as neuroimaging their data represent only correlates of the neuronal activity. Consequently, there is an increasing awareness that all noninvasive functional brain imaging methods are in fact subject to solving inverse problems to move from the measurement space to the source space in 
particular if the ultimate goal is to identify neuronal correlate of the measured hemodynamic and/or metabolic response.

Spatio-temporal resolution in the source space is a complex issue for all functional brain imaging methods, in particular, since some of them still do not report nor explore the problem at that level. Values reported for spatial and temporal resolution for a particular functional brain imaging method should be evaluated cautiously as the definitions differ across methods [1].

Furthermore, spatio-temporal source resolution is linked in all functional brain imaging methods. Closely spaced synchronously and even completely asynchronously activated cortical foci cannot be resolved by metabolic/ hemodynamic measures of neuronal activity. Consequently, reported excellent spatial resolution of fMRI in the millimeter scale and referring to the voxel size in the measurement space does not hold in the source space neither regarding the accuracy nor reliability [3] of a single functional focus nor as spatial resolution of the two nearby foci. It is surprising that the problem of fMRI spatial resolution in the source space remains undefined and unexplored as of now. Yet, fMRI has revolutionized the brain research and has contributed to a global acceptance of the noninvasive methods for studies of the human brain in basic and clinical applications.

MEG/EEG temporal resolution is reported to be on a millisecond scale compared to couple of seconds for fMRI and even $40 \mathrm{~s}$ for PET. Instantaneous magnetic fields generated predominantly by intra-neuronal ionic currents of the synchronously activated population of hundreds of thousands of neurons are often sampled at a rate of $1-5 \mathrm{kHz}$ leading to a millisecond and even sub-millisecond temporal resolution. In fMRI and PET it is the nature of the signal (i.e., hemodynamic and/or metabolic responses that lag $1 \mathrm{~s}$ after the fast neuronal events) and the time to build up the measured signals that lead to very slow temporal resolution of the two methods. The ability to measure the data rather quickly (up to $50 \mathrm{~ms}$ ) using fast sequences or the use of the fastest crystal detectors in PET do not contribute to improving the temporal resolution of the fMRI and PET, respectively. In all techniques, the ultimate limitation to the temporal resolution comes from the nature of the signal, not the hardware or software technical abilities.

Real time access to neuronal activity by the non-invasive EEG and MEG methods are most valuable and allow insight into what is currently considered as an emerging concept of multiple, hierarchically organized temporal scales in human brain functions [8]. Superb temporal resolution of MEG contributes to an improved spatial resolution. Numerical simulations demonstrated that the reverse holds as well. Only when all active sources are identified accurate time course estimation is possible [13].
Neurodynamic spatio-temporal data matrices of the measured data contain details on dynamic cortical networks and their functional connectivity that can be revealed using adequate source analysis and connectivity analysis approaches.

Spatio-temporal source localization relies on source modeling assumptions, inverse approaches, and statistical techniques. Unlike in fMRI, EEG and MEG researchers struggle between models and approaches which remain to be fully evaluated. Standardization is unlikely to occur as the choice of an experimental paradigm and research question of interest combined with the information-richness of the collected spatio-temporal neurodynamic data matrices is ultimately likely to guide a choice between nonlinear and linear approaches, focal and distributed source models, spatial filters, or probabilistic approaches. There is an increasing agreement that further advances in neurodynamic methods of EEG and MEG are linked to progress in critical evaluations of the existing spatio-temporal source localization programs and development of novel approaches.

Source localization based on EEG/ERP data is strongly affected by the volume conductor approximation and its conductive geometry profile. MRI-based realistic head models are developed. Most of the brain tissue is neither a good conductor nor a good isolator, and in the low frequency range exhibits mainly conductive properties with ions as charge carriers. The electrical characteristics of the impedance of many biological tissues are nonhomogenous, anisotropic, dispersive, and nonlinear. Head tissues, the skull, scalp, muscles, cerebrospinal fluid, and brain have different conductivities. One of the problems is to find the realistic values of conductivity and permittivity for each type of the tissue. Most of the values are measured for low intensity field values only. Values obtained by in vivo measurements should be preferred when possible. Determining the relevant discontinuities in the conductivity as well as getting accurate conductivity values for each of the sub-regions of the brain is challenging.

This Special Issue is related to the workshop organized within the framework of the COST NeuroMath Action whose main objective is to increase the knowledge on the mathematical methods able to estimate the cortical activity and connectivity in the human brain from non-invasive neurodynamic and hemodynamic measurements. NeuroMath is a COST Action in which scientists are called to harmonize their computational tools to offer a comprehensive approach to the problem of the estimation of brain activity and connectivity for sensory and cognitive tasks. The concept of brain connectivity is viewed as central for the understanding of the dynamic interactions between cortical regions. 
Structural and functional connectivity methods are increasingly in the focus of basic and clinical studies. In addition to experimental manipulations of functional brain connectivity using trans-cranial magnetic stimulation (TMS) computational approaches relying on structural and functional, in particular, neurodynamic, data can provide information on anatomical, statistical, functional, and effective brain connectivity. Neurodynamic insight into perception, cognition, social interactions, emotional processing, functional connectivity, and consciousness is expecting to lead, among others, to more efficient braincomputer interface, development of humanoid robots, and advances in neuroengineering. It is important, however, to emphasize that despite wide and increasing applications of the functional brain imaging methods their biggest strength lies in the fact that they all remain in a dynamic research phase. New era of functional brain imaging may be upon us as novel research problems open, as technical abilities to process and model the data develop and as we engage into gaining deeper insights into the advantages and limitations of each of the methods and keep revisiting concepts they rely on and remain open to new ones that are emerging.

The articles in this special issue cover a range of relevant topics that address most important problems and trust areas in the neurodynamic studies of functional connectivity and cognition such as spatio-temporal scales and links between electrical neuroimaging methodologies [6], methods for determining directed connectivity from multichannel data [4], face-activated neurodynamic cortical networks [14], experimental manipulation of the visual stimulus size and related increase in the spatial extent of the visual cortex in exploring potential biases in source location estimates in the primary visual cortex [11], spacetime network connectivity and cortical activations preceding Spike Wave Discharges in human absence epilepsy [7], combined imagery and SSVEP-based BCI control of an artificial upper limb [10], correlation of spectral EEG asymmetry with the experienced pleasantness of TV commercial advertisements [15], the use of Higuchi's fractal dimension for analysis of the effect of external periodic stressor on the brain oscillations and connectivity pattern [9], a novel method for recording neuronal depolarization and implications for imaging fast neuronal activity in the brain with electrical impedance tomography [12], and the effects of nicotine exposure on time-frequency distribution of VTA DA neurons' firing activities $[5]$.

Acknowledgment COST Action BM0601 workshop "Neurodynamic insight into functional connectivity, cognition, and consciousness" was held on March 27-28, 2010 in Dubrovnik, Croatia as a satellite event of the 17th International conference on biomagnetism-Biomag2010. We thank Professor Fabio Babiloni, the Chair of the NeuroMath COST Action BM0601, and the COST Office for their support.

\section{References}

1. Aine CJ (1995) A conceptual overview and critique of functional neuroimaging techniques in humans: I MRI/fMRI and PET. Crit Rev Neurobiol 9:229-309

2. Aine CJ (2010) Highlights of 40 years of SQUID-based brain research and clinical applications. In: Supek S, Susac A (eds) IFMBE Proceedings of the 17th international conference on biomagnetism advances in biomagnetism-biomag2010, vol 28. Springer, Heidelberg, pp 9-34

3. Bennett CM, Miller MB (2010) How reliable are the results from functional magnetic resonances imaging? Ann NY Acad Sci 1191:133-155

4. Blinowska KJ (2011) Review of the methods of determination of directed connectivity from multichannel data. Med Biol Eng Comput. doi:10.1007/s11517-011-0739-x

5. Chen T, Zhang D, Dragomir A, Akay YM, Akay M (2011) The effects of nicotine exposure and PFC transection on the timefrequency distribution of VTA DA neurons' firing activities. Med Biol Eng Comput. doi:10.1007/s11517-011-0759-6

6. Gonzalez Andino SL, Perrig S, Grave de Peralta Menendez R (2011) Spatiotemporal scales and links between electrical neuroimaging methodologies. Med Biol Eng Comput. doi: 10.1007/s11517-011-0769-4

7. Gupta D, Ossenblok P, van Luijtelaar G (2011) Space-time network connectivity and cortical activations preceding spike wave discharges in human absence epilepsy: a MEG study. Med Biol Eng Comput. doi:10.1007/s11517-011-0778-3

8. Hari R, Parkkonen L, Nangini C (2010) The brain in time: insights from neuromagnetic recordings. Ann NY Acad Sci 1191:89-109

9. Hinrikus H, Bachmann M, Karai D, Klonowski W, Lass J, Stepien P, Stepien R, Tuulik V (2011) Higuchi's fractal dimension for analysis of the effect of external periodic stressor on the brain electrical oscillations. Med Biol Eng Comput. doi: 10.1007/s11517-011-0768-5

10. Horki P, Solis Escalante T, Neuper C, Müller-Putz MP (2011) Combined motor imagery and SSVEP based BCI control of a 2 DoF artificial upper limb. Med Biol Eng Comput. doi: 10.1007/s11517-011-0750-2

11. Josef Golubic S, Susac A, Grilj V, Ranken D, Huonker R, Haueisen J, Supek S (2011) Size matters: MEG empirical and simulation study on source localization of the earliest visual activity in the occipital cortex. Med Biol Eng Comput. doi: 10.1007/s11517-011-0764-9

12. Oh T, Gilad O, Ghosh A, Schuettler M, Holder DS (2011) A novel method for recording neuronal depolarization with recording at $125-825 \mathrm{~Hz}$ : implications for imaging fast neural activity in the brain with electrical impedance tomography. Med Biol Eng Comput. doi:10.1007/s11517-011-0761-z

13. Supek S, Aine CJ (1997) Spatio-temporal modeling of neuromagnetic data: I. Multi source location versus time course estimation accuracy. Hum Brain Mapp 5:139-153

14. Susac A, Ilmoniemi RJ, Doug Ranken D, Supek S (2011) Face activated neurodynamic cortical networks. Med Biol Eng Comput. doi:10.1007/s11517-011-0740-4

15. Vecchiato V, Toppi J, Astolfi A, De Vico Fallani F, Cincotti F, Mattia D, Bez F, Babiloni F (2011) Spectral EEG frontal asymmetries correlate with the experienced pleasantness of TV commercial advertisements. Med Biol Eng Comput. doi: 10.1007/s11517-011-0747-x 